OPEN ACCESS

Edited by:

Zhenzhen Liu,

Sun Yat-sen University, China

Reviewed by:

Yanjun Hua,

Shanghai Jiao Tong University, China

Haoyu Chen,

Shantou University and the Chinese University of Hong Kong, China

*Correspondence:

Yijun $\mathrm{Hu}$

huyijun2014@163.com

Honghua Yu

yuhonghua@gdph.org.cn

Specialty section:

This article was submitted to

Ophthalmology,

a section of the journal

Frontiers in Medicine

Received: 29 June 2021 Accepted: 22 October 2021 Published: 18 November 2021

Citation:

Xu G, Wu G, Du Z, Zhu S, Guo Y, Yu H and Hu Y (2021) Distribution of White-to-White Corneal Diameter and Anterior Chamber Depth in Chinese Myopic Patients.

Front. Med. 8:732719.

doi: 10.3389/fmed.2021.732719

\section{Distribution of White-to-White Corneal Diameter and Anterior Chamber Depth in Chinese Myopic Patients}

\author{
Guihua $X u^{1}$, Guanrong $W u^{2}$, Zijing $D u^{2}$, Shanqing $Z h u^{3}$, Yunxiang Guo ${ }^{3}$, Honghua $Y u^{2 *}$ \\ and Yijun $\mathrm{Hu}^{2,3,4 *}$
}

${ }^{1}$ Department of Ophthalmology, Huizhou Municipal Central Hospital, Huizhou, China, ${ }^{2}$ Department of Ophthalmology, Guangdong Eye Institute, Guangdong Provincial People's Hospital, Guangdong Academy of Medical Sciences, Guangzhou, China, ${ }^{3}$ Aier Institute of Refractive Surgery, Refractive Surgery Center, Guangzhou Aier Eye Hospital, Guangzhou, China,

${ }^{4}$ Aier School of Ophthalmology, Central South University, Changsha, China

Purpose: To investigate the distribution of white-to-white (WTW) corneal diameter and anterior chamber depth (ACD) in Chinese myopia patients.

Methods: This was a cross-sectional observational study conducted at five ophthalmic centers. Anterior segment biometry was performed in 7,893 eyes of the 7,893 myopic patients using Pentacam, and the WTW and ACD were recorded. The distribution patterns of WTW and ACD were evaluated and the correlation between WTW and ACD was analyzed statistically.

Results: There were 4416 (55.95\%) males and 3477 (44.05\%) females. The age of the study population was $25.14 \pm 5.41$ years. Distribution of WTW was slightly positively skewed (Skewness $=0.0076$, Kurtosis $=0.3944$, KS $P=0.020$ ) with a mean of 11.65 $\pm 0.38 \mathrm{~mm}$ and a $95 \%$ normal range of $10.91-12.39 \mathrm{~mm}$. A significant difference in WTW was found among different myopia groups $(P<0.001)$. The ACD was normally distributed (Skewness $=0.899$, Kurtosis $=0.027, \mathrm{KS} P=0.086$ ). The mean ACD was $3.25 \pm 0.26 \mathrm{~mm}$ and the $95 \%$ normal range of was $2.74-3.75 \mathrm{~mm}$. A significant difference in ACD was also found among different myopia groups $(P=0.030)$. There was a significant correlation between WTW and ACD $(r=0.460, P<0.001)$.

Conclusions: In our study, 95\% of the Chinese myopic patients had a WTW within 10.91-12.39 mm and an ACD within 2.74-3.75 mm. ACD and WTW were significantly different among different myopia, gender and age groups. WTW was positively correlated with ACD.

Keywords: distribution, white-to-white corneal diameter, anterior chamber depth, Chinese, myopia patients

\section{INTRODUCTION}

Anterior segment parameters such as white-to-white (WTW) corneal diameter and anterior chamber depth (ACD) are not only of essential importance for preoperative evaluation of refractive surgery, but also provide crucial information about individual ocular anatomy (1-3). Accurate measurement of WTW and ACD is needed for implantable collamer lens (ICL) sizing before 
surgery (4). Implantation of an incorrect size ICL may lead to complications after the surgery, such as corneal endothelial damage, uveitis, and glaucoma (4). Besides, shallow central ACD was found to be an independent predictive risk factor for the development of any form of angle closure in the Handan Eye study (5) and in the Namil Study (6). Thus, it is important to identify such patients so that prophylactical treatment can be used to prevent an acute attack of angle-closure and primary angle-closure glaucoma (PACG). As for the WTW corneal diameter, an abnormal WTW may indicate corneal diseases like micro-cornea, microphthalmos, and so on (7). It is important to recognize these conditions before ocular surgery as they may be associated with other ocular and systemic disorders that compromise the outcomes of the surgery. WTW and ACD are also important parameters in some formulas used to calculate the power of intraocular lens (IOL) for cataract surgery $(8,9)$.

Previous studies have been carried out to investigate anterior segment parameters in various populations (10-13). However, results from these reports may not be directly applied to our patients due to possible ethnical differences in the anterior segment anatomy (1). In a recent study, the distribution of WTW was investigated in Chinese cataractous patients aged $63.7 \pm 12.4$ years (14). To date, little information is known about WTW and ACD distribution in young Chinese myopic adults, who represent the largest population of refractive surgery candidates in the world. In the present study, using pooled data from five ophthalmic centers we revealed the distribution patterns of WTW and ACD in this specific group of patients.

\section{MATERIALS AND METHODS}

\section{Participants}

A total of 7,893 eyes of 7,893 myopic patients from Guangzhou Aier Eye Hospital (GZ), Shenyang Aier Eye Hospital (SY), Chengdu Aier Eye Hospital (CD), Wuhan Aier Eye Hospital (WH), and Hankou Aier Eye Hospital (HK) were retrospectively recruited. The study was approved by the Institutional Review Board (IRB) of every hospital (GZ, SY, CD, WH, and HK) and conducted in agreement with the Declaration of Helsinki. The IRBs waived the need of informed consent as the study only involved review and analysis of medical records and no individual patient could be identified from the data (15, 16). Inclusion criteria were myopic patients with a spherical equivalent $(\mathrm{SE}) \leq-0.50 \mathrm{D}$ and good quality Scheimpflug scans. Only the right eyes of the patients were included for analysis. Exclusion criteria were coexisting corneal diseases, keratoconus, forme fruste keratoconus, severe dry eye, non-axial myopia (such as those caused by spherophakia), previous ocular trauma or surgery, uveitis, glaucoma, wearing contact lenses within the previous 2 weeks, age younger than 18 years (unstable refraction) or older than 40 years (to reduce the effects of the crystal lens on anterior chamber depth measurement) $(15,16)$.

\section{Examinations}

All of the eyes underwent routine ophthalmic examinations including decimal visual acuity, intraocular pressure (IOP), cycloplegic and manifest refraction, anterior segment examination by slit-lamp, corneal topography and tomography (Pentacam). Clinical data of the eyes were retrieved from an electronic medical record database. The spherical equivalent (SE) was defined as "spherical error $+1 / 2$ cylindrical error" using manifest refraction. The eyes were divided into four groups according to the manifest SE: low myopia $(-3.00 \mathrm{D}<\mathrm{SE} \leq-0.5$ $\mathrm{D}, \mathrm{LM})$, moderate myopia $(-6.00 \mathrm{D}<\mathrm{SE} \leq-3.00 \mathrm{D}, \mathrm{MM})$, high myopia $(-10.00 \mathrm{D}<\mathrm{SE} \leq-6.00 \mathrm{D}, \mathrm{HM})$ and extremely high myopia ( $\mathrm{SE} \leq-10 \mathrm{D}, \mathrm{EHM})$.

The WTW and ACD were measured with Pentacam by experienced technicians under dim light condition according to the standard procedures of the manufacturer (Oculus $\mathrm{GmbH}$, Wetzlar, Germany) as previously described $(15,16)$. The fixation target was set to a viewing distance of +1.00 to $0.00 \mathrm{D}$ (far vision) (17). Depth of anterior chamber (ACD) was the distance between the anterior surface of the crystalline lens and posterior surface of cornea, and WTW was the distance between nasal and temporal limbus points between the white sclera and the darker iris image (Figure 1). Repeated measurement was taken when Pentacam scans did not pass the quality check. Quality control and data retrieving of Pentacam examination were previously described $(15,16)$.

\section{Statistical Analysis}

Pooled data of the five ophthalmic centers were used for analysis. Distribution of the WTW and ACD was evaluated by Kolmogorov-Smirnov (KS) test. Data of WTW, ACD, age, and SE were presented as mean \pm standard deviation (SD). Kruskal-Wallis test was used for comparison of WTW and ACD among different myopia groups and age groups. Two sample $t$-test was used for ACD and WTW comparison between different genders among different myopia groups. Correlation between the WTW and ACD, between WTW/ACD and age, and between WTW/ACD and refractive error was evaluated by Spearman correlation test. $P<0.05$ was considered to be statistically significant.

\section{RESULTS}

\section{Demography}

There were 7,893 patients $(7,893$ eyes) included in the study and $55.95 \%$ of them were male. The mean age of the patients was $25.14 \pm 5.41$ years. The mean SE of the eyes was -4.87 $\pm 1.66 \mathrm{D}$. There was a significant difference in age, gender, SE, ACD, and WTW among patients from different ophthalmic centers (all $P<0.001$ ). Demographics of the eyes are shown in Table 1.

\section{Distribution of Corneal Diameter and Anterior Chamber Depth}

Distribution of WTW was slightly positively skewed (Figure 2; Skewness $=0.0076$, Kurtosis $=0.3944$, KS $P=0.020)$; the ACD was normally distributed (Figure 3; Skewness $=0.899$, Kurtosis $=0.027$, KS $P=0.086$ ). The mean WTW was $11.65 \pm$ $0.38 \mathrm{~mm}$ (95\% CI: $11.64-11.66)$ and the mean ACD of the study population was $3.25 \pm 0.26 \mathrm{~mm}$ (95\% CI 3.24-3.25). The $95 \%$ normal range of WTW in our study was $10.91-12.39 \mathrm{~mm}$, and 

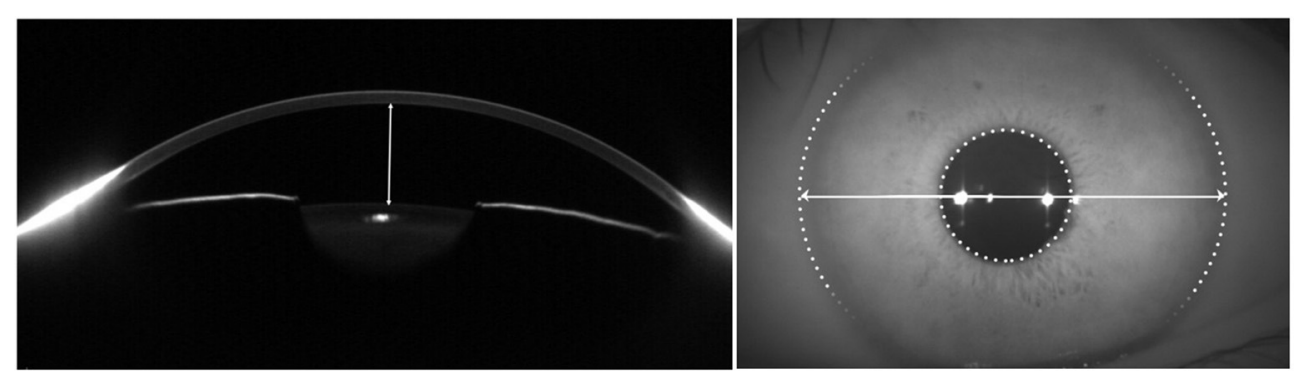

FIGURE 1 | Delineations of white-to-white (WTW) corneal diameter and anterior chamber depth (ACD) measurements with Pentacam. The distance between the anterior surface of the crystalline lens and posterior surface of cornea was ACD (left) and WTW was the distance between nasal and temporal limbus points between the white sclera (right).

TABLE 1 | Demographics of the subjects in different ophthalmic centers.

\begin{tabular}{|c|c|c|c|c|c|c|}
\hline & GZ & SY & CD & WH & HK & $P^{\dagger}$ \\
\hline Eyes (N) & 2340 & 2255 & 1480 & 1511 & 307 & $\mathrm{~N} / \mathrm{A}$ \\
\hline Eyes (\%) & $29.65 \%$ & $28.57 \%$ & $18.75 \%$ & $19.14 \%$ & $3.89 \%$ & $\mathrm{~N} / \mathrm{A}$ \\
\hline Male (\%) & $46.41 \%$ & $64.83 \%$ & $61.49 \%$ & $50.43 \%$ & $63.84 \%$ & $<0.0001$ \\
\hline Age (years) & $26.94 \pm 5.42$ & $23.88 \pm 5.14$ & $24.19 \pm 5.46$ & $25.39 \pm 5.03$ & $23.97 \pm 4.78$ & $<0.0001$ \\
\hline $\mathrm{ACD}(\mathrm{mm})^{\mathrm{a}}$ & $3.27 \pm 0.26$ & $3.29 \pm 0.26$ & $3.27 \pm 0.26$ & $3.21 \pm 0.24$ & $3.26 \pm 0.25$ & 0.0001 \\
\hline WTW (mm) ${ }^{\mathrm{a}}$ & $11.67 \pm 0.38$ & $11.67 \pm 0.36$ & $11.67 \pm 0.38$ & $11.63 \pm 0.38$ & $11.57 \pm 0.38$ & 0.0001 \\
\hline $\mathrm{SE}(\mathrm{D})^{\mathrm{a}}$ & $-5.17 \pm 2.23$ & $-4.81 \pm 1.71$ & $-5.27 \pm 2.23$ & $-5.28 \pm 1.93$ & $-5.65 \pm 2.68$ & 0.0001 \\
\hline
\end{tabular}

N, number of eyes; ACD, Anterior Chamber Depth; WTW, White-to-white; SE, Spherical Error; D, diopter; GZ, Guangzhou Aier Eye Hospital; SY, Shenyang Aier Eye Hospital; CD, Chengdu Aier Eye Hospital; WH, Wuhan Aier Eye Hospital; HK, Hankou Aier Eye Hospital; ${ }^{2}$ Presented as mean \pm standard deviation; ${ }^{\dagger}$ P-value for comparison among the five groups using Kruskal-Wallis test.

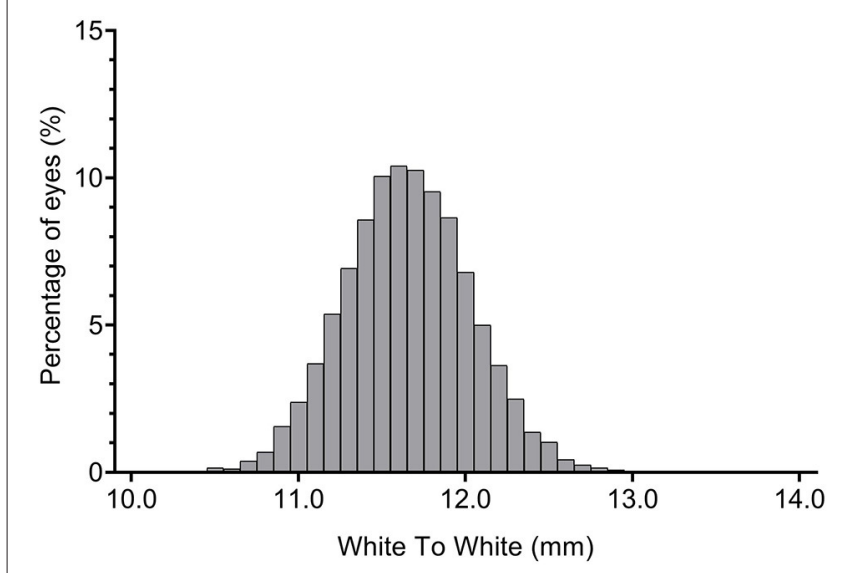

FIGURE 2 | Frequency distribution of white-to-white (WTW) corneal diameter.

the $95 \%$ normal range of ACD was $2.74-3.75 \mathrm{~mm}$. The smallest WTW was $10.2 \mathrm{~mm}$ and the WTW $<10.65 \mathrm{~mm}$ was seen in 25 eyes $(0.32 \%)$. The largest WTW was $13.3 \mathrm{~mm}$ and the WTW $>12.94 \mathrm{~mm}$ was seen in 3 eyes (0.04\%). Anterior chamber depth in our study population ranged from 2.07 to $4.35 \mathrm{~mm}$. There were 352 eyes (4.46\%) with an ACD $<2.8 \mathrm{~mm}$ and 889 eyes $(11.26 \%)$ more than $3.55 \mathrm{~mm}$.

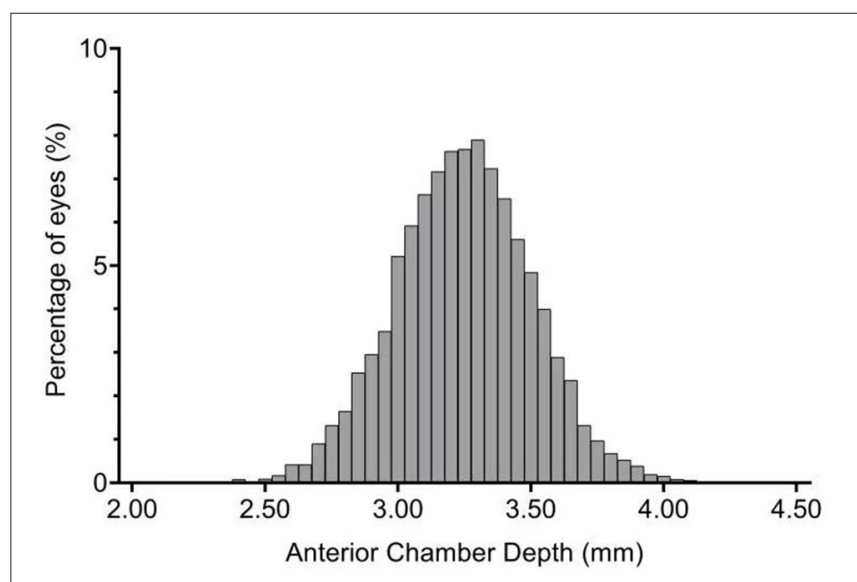

FIGURE 3 | Frequency distribution of anterior chamber depth (ACD).

Details about the WTW and ACD in different myopia groups are shown in Table 2. Significant differences in WTW and ACD were found among different myopia groups $(P<0.001$ and $P=$ 0.030 , respectively). Eyes in the LM group seemed to have slightly lower ACD but significantly larger WTW.

Comparison of WTW and ACD between female and male subjects in different myopia groups are shown in Table 3. 
TABLE 2 | Anterior chamber depth and white-to-white corneal diameter in different myopia groups of patients.

\begin{tabular}{|c|c|c|c|c|c|}
\hline Group & LM & MM & HM & EHM & $P$ \\
\hline N (\%) & 945 (11.97\%) & 4524 (57.32\%) & 2272 (28.8\%) & 152 (1.93\%) & / \\
\hline $\mathrm{ACD}(\mathrm{mm})^{\mathrm{a}}$ & $\begin{array}{c}3.22 \pm 0.25 \\
(2.73,3.72)\end{array}$ & $\begin{array}{l}3.25 \pm 0.26 \\
(2.74,3.75)\end{array}$ & $\begin{array}{l}3.25 \pm 0.25 \\
(2.75,3.75)\end{array}$ & $\begin{array}{l}3.25 \pm 0.27 \\
(2.73,3.78)\end{array}$ & 0.030 \\
\hline WTW $(\mathrm{mm})^{\mathrm{a}}$ & $\begin{array}{c}11.70 \pm 0.37 \\
(10.99,12.42)\end{array}$ & $\begin{array}{c}11.66 \pm 0.38 \\
(10.92,12.41)\end{array}$ & $\begin{array}{c}11.61 \pm 0.38 \\
(10.87,12.35)\end{array}$ & $\begin{array}{r}11.51 \pm 0.39 \\
(10.74,12.27)\end{array}$ & $<0.001$ \\
\hline $\mathrm{SE}(\mathrm{D})^{\mathrm{a}}$ & $\begin{array}{c}-2.27 \pm 0.49 \\
(-1.32,-3.23)\end{array}$ & $\begin{array}{c}-4.49 \pm 0.83 \\
(-2.87,-6.11)\end{array}$ & $\begin{array}{c}-7.08 \pm 0.89 \\
(-5.34,-8.82)\end{array}$ & $\begin{array}{c}-12.65 \pm 2.97 \\
(-6.83,-18.46)\end{array}$ & $<0.001$ \\
\hline
\end{tabular}

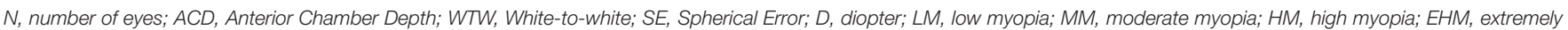

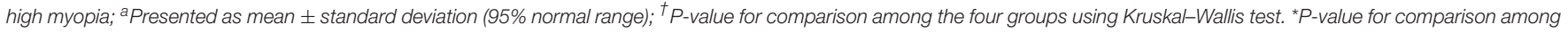
the five groups using one way ANOVA test.

TABLE 3 | Comparison of anterior chamber depth and white-to-white corneal diameter between different genders among different myopia groups.

\begin{tabular}{|c|c|c|c|c|c|c|c|c|}
\hline \multirow[t]{2}{*}{ Group } & \multicolumn{2}{|c|}{ LM } & \multicolumn{2}{|c|}{ MM } & \multicolumn{2}{|c|}{ HM } & \multicolumn{2}{|c|}{ EHM } \\
\hline & $\operatorname{ACD}(\mathrm{mm})^{\mathrm{a}}$ & WTW $(\mathrm{mm})^{\mathrm{a}}$ & $\operatorname{ACD}(\mathrm{mm})^{\mathrm{a}}$ & WTW (mm) ${ }^{a}$ & $\operatorname{ACD}(\mathrm{mm})^{\mathrm{a}}$ & WTW $(\mathrm{mm})^{\mathrm{a}}$ & $\operatorname{ACD}(\mathrm{mm})^{\mathrm{a}}$ & WTW $(\mathrm{mm})^{\mathrm{a}}$ \\
\hline Male & $3.26 \pm 0.24$ & $11.75 \pm 0.36$ & $3.31 \pm 0.25$ & $11.75 \pm 0.37$ & $3.31 \pm 0.25$ & $11.70 \pm 0.38$ & $3.29 \pm 0.30$ & $11.57 \pm 0.40$ \\
\hline Female & $3.10 \pm 0.26$ & $11.57 \pm 0.34$ & $3.16 \pm 0.25$ & $11.56 \pm 0.36$ & $3.19 \pm 0.25$ & $11.54 \pm 0.36$ & $3.23 \pm 0.24$ & $11.46 \pm 0.38$ \\
\hline$P$ & $<0.001^{\dagger}$ & $<0.001^{\dagger}$ & $<0.001^{\dagger}$ & $<0.001^{\dagger}$ & $<0.001^{\star}$ & $<0.001^{\dagger}$ & $0.082^{\dagger}$ & $0.038^{*}$ \\
\hline
\end{tabular}

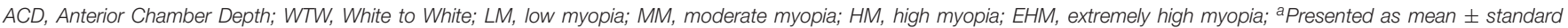
deviation. ${ }^{\dagger} P$-value for ACD and WTW comparison between different genders using two-sample $t$-test. ${ }^{*} P$-value for comparison among the five groups using Mann-whitney test.

TABLE 4 | Comparison of anterior chamber depth and white-to-white corneal diameter among different age groups.

\begin{tabular}{lcc}
\hline Age (years) & ${\text { ACD }(\mathbf{m m})^{\mathbf{a}}}$ & WTW $(\mathbf{m m})^{\mathbf{a}}$ \\
\hline $18-20$ & $3.34 \pm 0.23$ & $11.75 \pm 0.37$ \\
$21-23$ & $3.28 \pm 0.24$ & $11.68 \pm 0.38$ \\
$24-26$ & $3.25 \pm 0.25$ & $11.63 \pm 0.36$ \\
$27-29$ & $3.21 \pm 0.26$ & $11.60 \pm 0.37$ \\
$30-32$ & $3.16 \pm 0.25$ & $11.55 \pm 0.37$ \\
$33-35$ & $3.13 \pm 0.25$ & $11.55 \pm 0.35$ \\
$36-38$ & $3.11 \pm 0.27$ & $11.58 \pm 0.39$ \\
$39-40$ & $3.02 \pm 0.23$ & $11.55 \pm 0.38$ \\
$P^{\dagger}$ & $<0.001$ & $<0.001$ \\
\hline
\end{tabular}

ACD, Anterior Chamber Depth; WTW, White to White. ${ }^{\text {a }}$ Presented as mean \pm standard deviation. ${ }^{\dagger} P$-value for $A C D$ and WTW comparison between different age groups using Kruskal-Wallis test.

Significant larger WTW and ACD in males than females were observed in low, moderate and high myopia groups, and significant larger WTW but not ACD in male than female was observed in extremely high myopia group.

A significant difference was found among different age groups in terms of ACD and WTW measurements (all $P<0.001$ ). With increase of age, ACD was shallower and WTW was smaller as showed in Table 4.

No significant correlation was found between SE and ACD $(r$ $=-0.019, P=0.090)$, or between SE and WTW $(r=0.094, P$ $<0.001)$. A weak but statistically significant negative correlation was found between ACD and age $(r=-0.286, P<0.001)$, and between WTW and age $(r=-0.199, P<0.001)$.

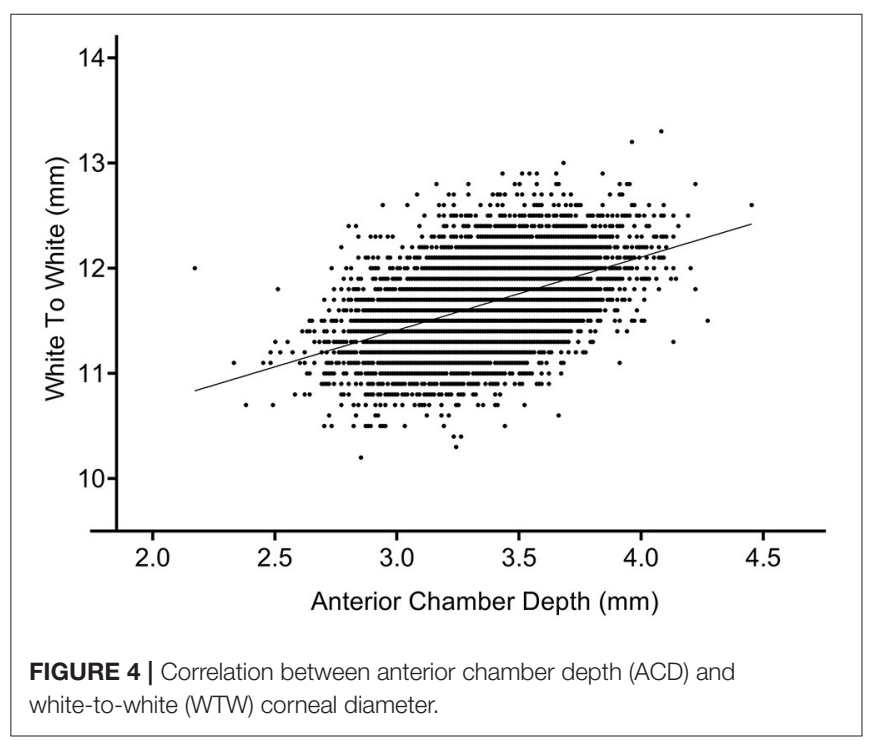

\section{Correlation Between WTW and ACD}

A significant positive correlation was found between WTW and ACD in our subjects $(r=0.460, P<0.001)$. Patients with shallower anterior chambers might also have smaller WTW (Figure 4).

\section{DISCUSSIONS}

The distribution patterns of WTW and ACD in myopic patients were investigated in the present multicenter study using 
pooled data from different ophthalmic centers of mainland China. We found that the average WTW was $11.65 \mathrm{~mm}$ and within $10.91-12.39 \mathrm{~mm}$ in $95 \%$ of eyes. The average ACD was $3.25 \mathrm{~mm}$, and $95.54 \%$ of eyes had an ACD of $2.74-3.75 \mathrm{~mm}$. Significant differences in ACD and WTW were found among different myopia groups. The WTW was increased in eyes with deeper ACD.

ICL implantation is a predictable and widely accepted surgery for the correction of myopia (18-20). Although the ICL surgery has been reported to be a relatively safe, effective, alterable, and even reversible surgical approach, a variety of possible complications are associated with the procedure, such as lens opacification, endothelial cell loss, high intraocular pressure, rotation of ICL, and anterior chamber inflammation $(7,21)$. Postoperative complications also include improper ICL vaulting due to incorrect ICL size selection, which is closely related to preoperative measurement of ACD and WTW (22). ICL sizing for ideal vaulting is challenging. A properly sized ICL can greatly help achieve an optimum vault after the surgery. Patients with a shallower ACD and a smaller WTW tend to have a lower postoperative vault. An over-low vault may increase the risk of lens opacification after surgery. On the contrary, patients with a deeper ACD and a larger WTW are more likely to have a higher postoperative vault. An excessively high vault may cause complications such as pupil ovalization, iritis, pigment dispersion, angle crowding, the liberation of inflammatory mediators, iris chaffing, endothelial cell loss, and angle-closure glaucoma (23). Thus, it is critical to determine the normal ranges of ACD and WTW in myopia eyes so that they can be used as references during ICL sizing.

Currently, the ICL sizes are available for eyes with an ACD of at least $2.80 \mathrm{~mm}$. In our study, $4.46 \%$ of eyes had an ACD $<2.80 \mathrm{~mm}$. In patients with high myopia and extremely high myopia, $3.59 \%$ of eyes had an ACD $<2.80 \mathrm{~mm}$. These patients may pose great challenges in ICL size selection. High myopia patients with a shallow ACD $(<2.80 \mathrm{~mm})$ are not rare (24). Although it was shown that high myopia patients with shallow ACD achieved satisfying and stable visual outcomes during a follow-up of $15.35 \pm 4.90$ months after ICL surgery, the longterm safety and stability require further investigation (25).

On the other hand, a shallow ACD is correlated with a higher risk of developing PACG (26). In western countries the majority of glaucoma is the open-angle type. However, the percentage of angle-closure glaucoma in the eastern population is almost $50 \%$ (1). The shallower ACD seen in the eastern population due to racial differences might be responsible for the discrepancy in PACG prevalence. Females have shallow ACD as compared to males in the eastern population putting them at higher risks of acute attack of angle-closure and PACG. In the Handan Eye study, 6,830 eligible subjects aged 30 years or older with open-angle were recruited and undergone gonioscopic examinations at baseline and follow-up visits; the results showed that shallow central ACD was a significant risk factor for development of any form of angle closure after 5year follow up $(O R=0.110, P=0.003)$ (5). In our study, ACD was significantly different among different myopia groups.
A previous study showed that a deeper ACD was related to thinner lenses in eyes with longer axial length, which was probably due to geometrical scaling during axial elongation of the eyes (27).

The average WTW in our study was $11.65 \pm 0.38 \mathrm{~mm}$, which was similar to Singh et al.'s study conducted in normal Indian subjects (WTW $=11.79 \pm 0.67 \mathrm{~mm}$ ) (1). In our study, the WTW was decreased in patients with the increase of myopia severity $(P$ $=0.030$ ), which was in accordance with the study conducted by Zha et al. (28). The decrease of WTW with higher myopia may be due to posterior traction of the limbus caused by elongation of the eyeball. However, further investigations are needed to reveal the exact mechanisms of this finding. Besides ACD, WTW is also of great importance for ICL sizing. Based on Visian ICL product information, patients with WTW $<10.65 \mathrm{~mm}$ may not be recommended for ICL implantation (24). In our study, $0.32 \%$ of myopia eyes had WTW measurement $<10.65 \mathrm{~mm}$. For these eyes, development of a new ICL size may be needed.

Both WTW and ACD are important parameters for IOL power selection in cataract surgery. ACD and WTW are indispensable biometric determinants for IOL power calculation formula, such as SRK-T, Holladay 2 and so on (9). Precise preoperative WTW and ACD measurement are significantly associated with accurate IOL positioning after cataract surgery, which is one of the important factors related to satisfactory postoperative visual outcomes $(8,9,29)$. In a recent study, larger WTW was observed in younger male patients and eyes with flatter corneas, deeper anterior chambers, thicker lenses, and thinner central corneal thickness (14). The largest WTW was found in eyes with axial length of 24.5 to $26 \mathrm{~mm}$ (14). Some of these findings seemed to be different from our results. One of the reasons might be that the populations were different in the two studies. While in Wei et al.'s study the participants were cataractous patients aged $63.7 \pm 12.4$ years, our study population was young myopic adults with a mean age of $25.14 \pm 5.41$ years. Different from our study, the WTW and ACD in Wei et al.'s study, might be affected by older age and the cataract (14).

We should be aware that the actual ACD and WTW values may vary according to different measuring instruments such as Orbscan, Pentacam, or IOL Master because of different measuring principles of these instruments. The ACD and WTW measurements of Pentacam may not be interchangeable with Orbscan or IOL Master (30). Although other parameters such as sulcus-to-sulcus (STS) distance on ultrasound biomicroscopy also can be used to calculate the ICL size (31). WTW and ACD were the two major parameters recommended by the manufacturer for choosing ICL size (Visian ICL Product Information: Visian ICL For Myopia. Available at http://www. accessdata.fda.gov/cdrh_docs/pdf3/p030016c.pdf).

What cannot be ignored is that patients with small WTW and narrow ACD may have a higher risk of low ICL vault after surgery (23). In our study, the normal range of ACD was 2.74$3.75 \mathrm{~mm}$, and WTW was $10.91-12.39 \mathrm{~mm}$, indicating a relatively large range of ACD and WTW measurements in myopia eyes. The variety in ACD and WTW may lead to multiple choices in ICL size, and sometimes it is challenging to determine the 
most appropriate ICL size. Moreover, it remains undetermined about what is a safe ICL vault after surgery. Several studies have reported that $90 \mu \mathrm{m}$ is the minimum safe vault $(32,33)$. Gonvers et al. recommended a central vault of $150 \mu \mathrm{m}$, to protect the lens from contact with the ICL (34). Choi et al. reported an ideal ICL vault to be $250-750 \mu \mathrm{m}$ (35). The maximum safe vault may be associated with preoperative ACD. A high preoperative ACD is likely to render a high vault after ICL implantation, and may have better tolerance for high ICL vault postoperatively. However, an ideal ACD which could predictive a safe range of ICL vault is still under investigation (36). Importantly, it is necessary to take into accounts the effects of accommodation on vaulting and the age-related reduction of the central vault when selecting the ideal ICL size.

Our study has some limitations. Firstly, we only included myopic patients in the study. Distribution of ACD and WTW in emmetropic and hyperopic subjects needs to be determined in future investigations. Secondly, our results can only be applied to a relatively young age group (18-40 years). In older patients the distribution pattern of ACD is significantly different, although the difference in WTW with aging may be less significant. Thirdly, we did not collect axial length (AL) data, and the relationship between AL and ACD/WTW could not be assessed in our study. Since the AL is better indicator of growth of the eye in myopia compared to the SE, it is important to evaluate the ACD and WTW distribution in eyes with different AL. Lastly, the study subjects in the five ophthalmic centers were inhomogeneous in terms of age and gender which might have some impact on the results, but we believe that the current results maybe more representative of the WTW and ACD in a real world scenario, instead of a specific "uniform" population.

In conclusion, we demonstrated the distribution patterns of ACD and WTW in Chinese myopic patients using multicenter data. The 95\% normal range for ACD was $2.74-3.75 \mathrm{~mm}$ and 10.91-12.39 mm for WTW. ACD and WTW were significantly different among different myopia, gender and age groups. WTW was positively correlated with ACD.

\section{REFERENCES}

1. Singh K, Gupta S, Moulick PS, Bhargava N, Sati A, Kaur G, et al. Study of distribution of white-to-white corneal diameter and anterior chamber depth in study population obtained with optical biometry using intraocular lens (IOL) master. Med J Armed Forces India. (2019) 75:400-5. doi: 10.1016/j.mjafi.2018.06.001

2. Reinstein DZ, Gobbe MTJ. Archer: anterior segment biometry: a study and review of resolution and repeatability data. $J$ Refract Surg. (2012) 28:509-20. doi: 10.3928/1081597X-201206 20-02

3. Shankar H, Taranath D, Santhirathelagan CT, Pesudovs K. Anterior segment biometry with the Pentacam: comprehensive assessment of repeatability of automated measurements. J Cataract Refract Surg. (2008) 34:103-13. doi: 10.1016/j.jcrs.2007.09.013

4. Wan $\mathrm{T}$, Yin $\mathrm{H}$, Yang $\mathrm{Y}, \mathrm{Wu} \mathrm{F}, \mathrm{Wu} \mathrm{Z}$, Yang $\mathrm{Y}$. Comparative study of anterior segment measurements using 3 different instruments in myopic patients after ICL implantation. BMC Ophthalmol. (2019) 19:182. doi: 10.1186/s12886-019-1194-y

\section{DATA AVAILABILITY STATEMENT}

The raw data supporting the conclusions of this article will be made available by the authors, without undue reservation.

\section{ETHICS STATEMENT}

The study was approved by the Institutional Review Board (IRB) of every hospital (GZ, SY, CD, WH, and HK) and conducted in agreement with the Declaration of Helsinki. Written informed consent for participation was not required for this study in accordance with the national legislation and the institutional requirements.

\section{AUTHOR CONTRIBUTIONS}

GX and YH designed the study and wrote the manuscript. GX, GW, ZD, SZ, and YG organized and analyzed the data and commented on the manuscript. HY and YH supervised the study and edited the manuscript. All authors contributed to the article and approved the submitted version.

\section{FUNDING}

This work was supported by Grant A2021378 from the Medical Scientific Research Foundation of Guangdong Province, China (YH), Grant 2018SK50106 from the Technology Innovation Guidance Program of Hunan Province (YH), Grant AM1909D2 and AR1909D2 from the Science Research Foundation of Aier Eye Hospital Group (YH).

\section{ACKNOWLEDGMENTS}

We thank the following doctors for providing the Pentacam data: Xuejun Fang and Jia Liu from SY, Jin Zhou and Fangfang Li from CD, Qingsong Zhang and Na Huang from WH, Xiaohua Lei and Li Jiang from HK.

5. Zhang Y, Zhang Q, Thomas R, Li SZ, Wang NL. Development of angle closure and associated risk factors: the Handan eye study. Acta Ophthalmol. (2021) 1-9. doi: 10.1111/aos.14887

6. Kim YY, Lee JH, Ahn MD, Kim CY, Namil Study Group, Korean Glaucoma Society. Angle closure in the Namil study in central South Korea. Arch Ophthalmol. (2012) 130:1177-83. doi: 10.1001/archophthalmol.20 12.1470

7. Alfonso JF, Lisa C, Fernandez-Vega L, Almanzar D, Perez-Vives C, MontésMicó R. Prevalence of cataract after collagen copolymer phakic intraocular lens implantation for myopia, hyperopia, and astigmatism. J Cataract Refract Surg. (2015) 41:800-5. doi: 10.1016/j.jcrs.2014.07.039

8. Wang Z, Yang W, Li D, Chen W, Zhao Q, Li Y, et al. Evaluation and comparison of a novel Scheimpflug-based optical biometer with standard partial coherence interferometry for biometry and intraocular lens power calculation. Exp Ther Med. (2021) 21:326. doi: 10.3892/etm.2021.9757

9. Hipolito-Fernandes D, Luis ME, Serras-Pereira R, Gil P, Maduro V, Feijao J, et al. Anterior chamber depth, lens thickness and intraocular lens calculation formula accuracy: nine formulas comparison. Br J Ophthalmol. (2020). 0:1-7. doi: 10.1136/bjophthalmol-2020-317822 
10. Baumeister M, Terzi E, Ekici Y, Kohnen T. Comparison of manual and automated methods to determine horizontal corneal diameter. J Cataract Refract Surg. (2004) 30:374-80. doi: 10.1016/j.jcrs.2003.06.004

11. Hashemi H, KhabazKhoob M, Yazdani K, Mehravaran S, Mohammad K, Fotouhi A. White-to-white corneal diameter in the Tehran Eye Study. Cornea. (2010) 29:9-12. doi: 10.1097/ICO.0b013e3181a9d0a9

12. Rufer F, Schroder A, Erb C. White-to-white corneal diameter: normal values in healthy humans obtained with the Orbscan II topography system. Cornea. (2005) 24:259-61. doi: 10.1097/01.ico.0000148312.01805.53

13. Elbaz U, Barkana Y, Gerber Y, Avni I, Zadok D. Comparison of different techniques of anterior chamber depth and keratometric measurements. Am J Ophthalmol. (2007) 143:48-53. doi: 10.1016/j.ajo.2006.08.031

14. Wei L, He W, Meng J, Qian D, Lu Y, Zhu X. Evaluation of the white-to-white distance in 39,986 Chinese cataractous eyes. Invest Ophthalmol Vis Sci. (2021) 62:7. doi: 10.1167/iovs.62.1.7

15. Hu Y, Zhu S, Xiong L, Fang X, Liu J, Zhou J, et al. A multicenter study of the distribution pattern of posterior corneal astigmatism in Chinese myopic patients having corneal refractive surgery. Sci Rep. (2020) 10:16151. doi: 10.1038/s41598-020-73195-w

16. Xu G, Hu Y, Zhu S, Guo Y, Xiong L, Fang X, et al. A multicenter study of interocular symmetry of corneal biometrics in Chinese myopic patients. Sci Rep. (2021) 11:5536. doi: 10.1038/s41598-021-84937-9

17. Domínguez-Vicent A, Monsálvez-Romín D, Del Águila-Carrasco AJ, FerrerBlasco T, Montés-Micó R. Changes in the anterior chamber during accommodation assessed with a Scheimpflug system. J Cataract Refract Surg. (2014) 40:1790-7. doi: 10.1016/j.jcrs.2014.02.043

18. Igarashi A, Shimizu K, Kamiya K. Eight-year follow-up of posterior chamber phakic intraocular lens implantation for moderate to high myopia. Am J Ophthalmol. (2014) 157:532-9 e1. doi: 10.1016/j.ajo.2013.11.006

19. Sanders DR, Vukich JA, Doney K, Gaston M, Implantable Contact Lens in Treatment of Myopia Study Group. U.S. Food and Drug Administration clinical trial of the Implantable Contact Lens for moderate to high myopia. Ophthalmology. (2003) 110:255-66. doi: 10.1016/S0161-6420(02)01771-2

20. Kamiya K, Igarashi A, Shimizu K, Matsumura K, Komatsu M. Visual performance after posterior chamber phakic intraocular lens implantation and wavefront-guided laser in situ keratomileusis for low to moderate myopia. Am J Ophthalmol. (2012) 153:1178-86 e1. doi: 10.1016/j.ajo.2011.12.005

21. Goukon H, Kamiya K, Shimizu K, Igarashi A. Comparison of corneal endothelial cell density and morphology after posterior chamber phakic intraocular lens implantation with and without a central hole. $\mathrm{Br} J$ Ophthalmol. (2017) 101:1461-5. doi: 10.1136/bjophthalmol-2016-309363

22. Guber I, Mouvet V, Bergin C, Perritaz S, Othenin-Girard P, Majo F. Clinical Outcomes and cataract formation rates in eyes 10 Years after posterior phakic lens implantation for myopia. JAMA Ophthalmol. (2016) 134:487-94. doi: 10.1001/jamaophthalmol.2016.0078

23. Lim DH, Lee MG, Chung ES, Chung TY. Clinical results of posterior chamber phakic intraocular lens implantation in eyes with low anterior chamber depth. Am J Ophthalmol. (2014) 158:447-54 e1. doi: 10.1016/j.ajo.2014. 06.005

24. Wang YE, Li Y, Wang D, He M, Lin S. Comparison of factors associated with occludable angle between american Caucasians and ethnic Chinese. Invest Ophthalmol Vis Sci. (2013) 54:7717-23. doi: 10.1167/iovs.1312850

25. Niu L, Miao H, Han T, Ding L, Wang X, Zhou X. Visual outcomes of Visian ICL implantation for high myopia in patients with shallow anterior chamber depth. BMC Ophthalmol. (2019) 19:121. doi: 10.1186/s12886-019-1 $132-\mathrm{z}$
26. Devereux JG, Foster PJ, Baasanhu J, Uranchimeg D, Lee PS, Erdenbeleig T, et al. Anterior chamber depth measurement as a screening tool for primary angle-closure glaucoma in an East Asian population. Arch Ophthalmol. (2000) 118:257-63. doi: 10.1001/archopht.118.2.257

27. Muralidharan G, Martinez-Enriquez E, Birkenfeld J, Velasco-Ocana M, PerezMerino P, Marcos S. Morphological changes of human crystalline lens in myopia. Biomed Opt Express. (2019) 10:6084-95. doi: 10.1364/BOE.10.006084

28. Zha Y, Feng W, Han X, Cai J. Evaluation of myopic corneal diameter with the Orbscan II Topography System. Graefes Arch Clin Exp Ophthalmol. (2013) 251:537-41. doi: 10.1007/s00417-012-2069-6

29. Plat J, Hoa D, Mura F, Busetto T, Schneider C, Payerols A, et al. Clinical and biometric determinants of actual lens position after cataract surgery. $J$ Cataract Refract Surg. (2017) 43:195-200. doi: 10.1016/j.jcrs.2016.11.043

30. Packer M. Meta-analysis and review: effectiveness, safety, and central port design of the intraocular collamer lens. Clin Ophthalmol. (2016) 10:1059-77. doi: 10.2147/OPTH.S111620

31. Kojima T, Yokoyama S, Ito M, Horai R, Hara S, Nakamura T, et al. Optimization of an implantable collamer lens sizing method using highfrequency ultrasound biomicroscopy. Am J Ophthalmol. (2012) 153:632-7, 637.e1. doi: 10.1016/j.ajo.2011.06.031

32. Dougherty PJ, Rivera RP, Schneider D, Lane SS, Brown D, Vukich J. Improving accuracy of phakic intraocular lens sizing using highfrequency ultrasound biomicroscopy. J Cataract Refract Surg. (2011) 37:13-8. doi: $10.1016 /$ j.jcrs.2010.07.014

33. Kamiya K, Shimizu K, Komatsu M. Factors affecting vaulting after implantable collamer lens implantation. J Refract Surg. (2009) 25:259-64. doi: 10.3928/1081597X-20090301-04

34. Gonvers M, Bornet C, Othenin-Girard P. Implantable contact lens for moderate to high myopia: relationship of vaulting to cataract formation. $J$ Cataract Refract Surg. (2003) 29:918-24. doi: 10.1016/S0886-3350(03)00065-8

35. Choi KH, Chung SE, Chung TY, Chung ES. Ultrasound biomicroscopy for determining visian implantable contact lens length in phakic IOL implantation. J Refract Surg. (2007) 23:362-7. doi: 10.3928/1081-597X-20070401-08

36. Seo JH, Kim MK, Wee WR, Lee JH. Effects of white-to-white diameter and anterior chamber depth on implantable collamer lens vault and visual outcome. J Refract Surg. (2009) 25:730-8. doi: 10.3928/1081597X-20090707-08

Conflict of Interest: The authors declare that the research was conducted in the absence of any commercial or financial relationships that could be construed as a potential conflict of interest.

Publisher's Note: All claims expressed in this article are solely those of the authors and do not necessarily represent those of their affiliated organizations, or those of the publisher, the editors and the reviewers. Any product that may be evaluated in this article, or claim that may be made by its manufacturer, is not guaranteed or endorsed by the publisher.

Copyright (c) $2021 \mathrm{Xu}, \mathrm{Wu}, \mathrm{Du}, \mathrm{Zhu}, \mathrm{Guo}, \mathrm{Yu}$ and Hu. This is an open-access article distributed under the terms of the Creative Commons Attribution License (CC BY). The use, distribution or reproduction in other forums is permitted, provided the original author(s) and the copyright owner(s) are credited and that the original publication in this journal is cited, in accordance with accepted academic practice. No use, distribution or reproduction is permitted which does not comply with these terms. 